Bull. Korean Math. Soc. 50 (2013), No. 5, pp. 1683-1691

http://dx.doi.org/10.4134/BKMS.2013.50.5.1683

\title{
ON WARPED PRODUCT SPACES WITH A CERTAIN RICCI CONDITION
}

\author{
Byung Hak Kim, Sang Deok Lee, Jin Hyuk Choi, and Young Ok Lee
}

\begin{abstract}
In this paper, we obtain the criteria that the Riemannian manifold $B$ is Einstein or a gradient Ricci soliton from the information of the second derivative of $f$ in the warped product space $R \times_{f} B$ with gradient Ricci solitons. Moreover, we construct new examples of nonEinstein gradient Ricci soliton spaces with an Einstein or non-Einstein gradient Ricci soliton leaf using our main theorems. Finally we also get analogous criteria for the Lorentzian version.
\end{abstract}

\section{Introduction}

In [5], R. S. Hamilton introduced the concept of Ricci solitons, which are special solutions of the Ricci flow of Hamilton. Recently the study of gradient Ricci solitons has become increasing and exhibits rich geometric properties.

A Riemannian metric $g$ on a complete Riemannian manifold $M$ is called a Ricci soliton if there exists a smooth vector field $X$ such that the Ricci tensor satisfies the following equation

$$
R i c+\frac{1}{2} \mathfrak{L}_{X} g=\rho g
$$

for some constant $\rho$, where $\mathfrak{L}_{X}$ is the Lie derivative with respect to $X([2,3$, $4,7,10,11])$.

The Ricci soliton is called shrinking if $\rho>0$, steady if $\rho=0$ and expanding if $\rho<0$. If $X=\nabla h$ for some function $h$ on $M$, then $M$ is called a gradient Ricci soliton ([10]). In this case the equation (1.1) can be rewritten as

$$
\text { Ric }+ \text { Hess } h=\rho g \text {. }
$$

It is well known that when $\rho \leq 0$ all compact solitons are necessarily Einstein $([4])$, and a Ricci soliton on a compact manifold has a constant curvature in dimension $2([5])$ as well as in dimension $3([6])$. Moreover a Ricci soliton on

Received December 4, 2012.

2010 Mathematics Subject Classification. Primary 53C25, 53B21.

Key words and phrases. Ricci curvature, Einstein metric, warped product space.

The first author was supported by the Research Grant 2011 of Kyung Hee University (KHU-20110480). 
a compact manifold is a gradient Ricci soliton ([7]), and a compact shrinking soliton is always gradient ([9]).

On the other hand, in the noncompact case, Perelman ([9]) has studied and classified the 3-dimensional shrinking gradient Ricci solitons with bounded nonnegative sectional curvature.

Since the concept of Ricci solitons is a natural extension of an Einstein manifold, it is meaningful to construct a non-Einstein gradient Ricci soliton and study Riemannian product spaces or warped product spaces with a gradient Ricci soliton. Indeed, for the study of Ricci soliton and construction of various Ricci solitons, not only the relationship between the base space $B$ and the total space $R^{p} \times B$ or $R \times_{f} B$ with gradient Ricci solitons, but also criteria whether $B$ is an Einstein or non-Einstein gradient Ricci soliton in $R \times_{f} B$ are very useful. Such relationships or criteria will give methods of the construction of model space with non-Einstein gradient Ricci soliton which has an Einstein or non-Einstein gradient Ricci soliton leaf.

In this paper, we review that the Riemannian product space $M=R^{p} \times B$ is a gradient Ricci soliton if and only if $B$ is a gradient Ricci soliton. Furthermore, we obtain that, in the warped product space $M=R \times_{f} B$ with a gradient Ricci soliton, $B$ is Einstein (or non-Einstein gradient Ricci soliton) if the second derivative of $f$ is non-vanishing (or vanishing, respectively).

Using our main theorems, we can construct new examples of non-Einstein gradient Ricci soliton spaces with an Einstein or non-Einstein gradient Ricci soliton leaf.

Finally we consider the Lorentzian warped product spaces $R \times_{f} B$ with gradient Ricci solitons and obtain criteria for $B$ to be Einstein or a gradient Ricci soliton, and find examples.

\section{Gradient Ricci solitons in Riemannian product spaces}

Let $(B, g)$ be an $n$-dimensional Riemannian manifold with a Riemannian metric $g$ and let $M=R \times B$ be the product Riemannian manifold with the Riemannian metric $\tilde{g}=\left(\begin{array}{ll}1 & 0 \\ 0 & g\end{array}\right)$. Then the curvature tensors $\tilde{K}$ and $K$ of $M$ and $B$, respectively, are given by $\tilde{K}_{d c b}{ }^{a}=K_{d c b}{ }^{a}$ and the others are zero, where the range of indices $a, b, c, \ldots$ is $\{2,3, \ldots, n+1\}$. Hence the Ricci curvature tensors $\tilde{S}$ and $S$ of $M$ and $B$, respectively, are given by $\tilde{S}_{a b}=S_{a b}$ and the others are zero. The scalar curvatures $\tilde{r}$ and $r$ of $M$ and $B$, respectively, are related by $\tilde{r}=r$.

Suppose that $B$ is a gradient Ricci soliton. Then $S_{a b}=\rho g_{a b}-\nabla_{a} k_{b}$ for some smooth function $k: B \rightarrow R$ and a constant $\rho$ on $B$.

Take $h: R \times B \rightarrow R$ such that $h\left(t, v_{2}, \ldots, v_{n+1}\right)=\frac{\rho}{2} t^{2}+k\left(v_{2}, \ldots, v_{n+1}\right)$ for all $\left(t, v_{2}, \ldots, v_{n+1}\right)$ in $R \times B$. Then we get $\tilde{S}_{a b}=S_{a b}=\rho g_{a b}-\nabla_{a} k_{b}=$ $\rho \tilde{g}_{a b}-\widetilde{\nabla}_{a} \widetilde{\nabla}_{b} h, \tilde{S}_{a 1}=0=-\widetilde{\nabla}_{a} \widetilde{\nabla}_{1} h, \tilde{S}_{11}=0=\rho-\widetilde{\nabla}_{1} \widetilde{\nabla}_{1} h$, where the indices $a, b, c, \ldots$ run over the range $\{2, \ldots, n+1\}$. Thus we see that if $B$ is a gradient Ricci soliton, then $R \times B$ is a gradient Ricci soliton. 
Conversely, if we suppose that $R \times B$ is a gradient Ricci soliton, then $\tilde{S}_{i j}=$ $\rho \tilde{g}_{i j}-\widetilde{\nabla}_{i} \widetilde{\nabla}_{j} k$ for some smooth function $k: R \times B \rightarrow R$ and for some constant function $\rho$ on $R \times B$. So we get

$$
S_{a b}=\rho g_{a b}-\nabla_{a} \nabla_{b} k, \quad \partial_{1} k_{a}=0, \quad \rho-\partial_{1} k_{1}=0 .
$$

From (2.1), we see that $k$ and $\rho$ are of the forms

$$
k=k\left(t, v_{a}\right)=m(t)+\ell\left(v_{a}\right), \quad m(t)=\frac{1}{2} \rho t^{2}+\mu t+\nu
$$

for $t \in R,\left(v_{a}\right)=\left(v_{2}, \ldots, v_{n+1}\right) \in B$ and constants $\mu, \nu$.

Since $k\left(t, v_{a}\right)=\left(\frac{1}{2} \rho t^{2}+b t+c\right)+\ell\left(v_{a}\right)$, we get $S_{a b}=\rho g_{a b}-\nabla_{a} \nabla_{b} \ell$. Hence $B$ is a gradient Ricci soliton. So, we can state if $R \times B$ is a gradient Ricci soliton, then $B$ is a gradient Ricci soliton. Therefore we see that the Riemannian product space $R \times B$ is a gradient Ricci soliton if and only if $B$ is a gradient Ricci soliton.

Next consider the Riemannian product space $M=R^{p} \times B$. Then we have

$$
\tilde{K}_{d c b}{ }^{a}=K_{d c b}{ }^{a}, \quad \tilde{S}_{a b}=S_{a b}, \quad \tilde{r}=r
$$

and the others are zero, where the range of indices $a, b, c, \ldots$ is $\{p+1, p+$ $2, \ldots, p+n\}$, and $\tilde{K}, \tilde{S}, \tilde{r}(K, S, r$ respectively) are Riemannian curvature tensor, Ricci curvature tensor and scalar curvature of $M(B$ respectively).

Suppose that $B$ is a gradient Ricci soliton. Then $S_{a b}=\rho g_{a b}-\nabla_{a} k_{b}$ for some smooth function $k: B \rightarrow R$ and a constant $\rho$ on $B$.

Take a function $h: R^{p} \times B \rightarrow R$ defined by

$$
h\left(u_{x}, v_{a}\right)=\frac{\rho}{2}\left(\left(u_{1}\right)^{2}+\left(u_{2}\right)^{2}+\cdots+\left(u_{p}\right)^{2}\right)+k\left(v_{a}\right)
$$

for all $\left(u_{x}, v_{a}\right)=\left(u_{1}, u_{2}, \ldots, u_{p}, v_{p+1}, v_{p+2}, \ldots, v_{p+n}\right)$ in $R^{p} \times B$. Then we obtain

$$
\tilde{S}_{a b}=\rho \tilde{g}_{a b}-\widetilde{\nabla}_{a} \widetilde{\nabla}_{b} h, \tilde{S}_{a x}=\rho \tilde{g}_{a x}-\widetilde{\nabla}_{a} \widetilde{\nabla}_{x} h, \tilde{S}_{x y}=\rho \tilde{g}_{x y}-\widetilde{\nabla}_{x} \widetilde{\nabla}_{y} h
$$

due to (2.3) and (2.4). Hence we see that if $B$ is a gradient Ricci soliton, then $R^{p} \times B$ is a gradient Ricci soliton. Since the standard $n$-sphere $S^{n}$ is a gradient Ricci soliton, applying this fact, $R^{p} \times S^{n}$ is a gradient Ricci soliton.

Suppose that $R^{p} \times B$ is a gradient Ricci soliton. Then $\tilde{S}_{i j}=\rho \tilde{g}_{i j}-\widetilde{\nabla}_{i} \widetilde{\nabla}_{j} k$ for some smooth function $k: R^{p} \times B \rightarrow R$ and for some constant function $\rho$ on $R^{p} \times B$, where the range of indies $i, j, k, \ldots$ is $\{1,2, \ldots, p+n\}$. Using (2.3), we obtain

$$
S_{a b}=\rho g_{a b}-\nabla_{a} \nabla_{b} k, \quad \partial_{x} k_{a}=0, \quad \rho \delta_{x y}-\partial_{x} k_{y}=0,
$$

where the range of indices $x, y, z, \ldots$ is $\{1,2, \ldots, p\}$. From $(2.6)_{2}$, and $(2.6)_{3}$, we see that $k\left(u_{x}, v_{a}\right)=m\left(u_{x}\right)+\ell\left(v_{a}\right)$ for some smooth function $\ell$ on $B$ and $m\left(u_{x}\right)=\frac{\rho}{2} \sum_{x=1}^{p}\left(u_{x}\right)^{2}$. Hence we can see that $\nabla_{a} \nabla_{b} k=\nabla_{a} \nabla_{b} \ell, S_{a b}=\rho g_{a b}-$ $\nabla_{a} \nabla_{b} \ell$. Therefore we see that if $R^{p} \times B$ is a gradient Ricci soliton, then $B$ is a gradient Ricci soliton. 
From this fact and upper result, we see that if $B$ is a gradient Ricci soliton if and only if $R^{p} \times B$ is a gradient Ricci soliton.

In [10], P. Petersen and W. Wylie proved that:

Theorem 2.1. If a gradient soliton splits $(M, g)=\left(M_{1} \times M_{2}, g_{1}+g_{2}\right)$ as a Riemannian product, then $f\left(x_{1}, x_{2}\right)=f_{1}\left(x_{1}\right)+f_{2}\left(x_{2}\right)$ also splits in such a way that each $\left(M_{i}, g_{i}, f_{i}\right)$ is a soliton Ric $g_{i}+$ Hess $_{i}=\lambda g_{i}$.

In connection with Theorem 2.1, we can prove that:

Theorem 2.2. Let $B \times F$ be a gradient Ricci soliton for a function $h(p, q)=$ $h_{1}(p)+h_{2}(q)$ for $(p, q) \in B \times F$. Then $B$ and $F$ are gradient Ricci solitons for $h_{1}$ and $h_{2}$, respectively.

Proof. From assumptions, we have

$$
\widetilde{S}_{i j}+\widetilde{\nabla}_{i} \widetilde{\nabla}_{j} h=\rho \widetilde{g}_{i j}
$$

for some constant $\rho$. Then we can see that

$$
S_{a b}+\nabla_{a} \nabla_{b} h_{1}=\rho g_{a b}, \quad \bar{S}_{x y}+\bar{\nabla}_{x} \bar{\nabla}_{y} h_{2}=\rho \bar{g}_{x y},
$$

that is, $B$ and $F$ are gradient Ricci solitons for $h_{1}$ and $h_{2}$, respectively.

From the relations of the (2.7) and (2.8), we can get the converse of the Theorem 2.1 .

Theorem 2.3. If $B$ and $F$ are gradient solitons for function $h_{1}$ and $h_{2}$, and constants $\lambda=\mu$ respectively, then $B \times F$ is a gradient Ricci soliton for $h_{1}+h_{2}$ and $\rho=\lambda=\mu$.

\section{Gradient Ricci solitons in warped product spaces}

Consider the warped product space $M=R \times{ }_{f} B$ with $\tilde{g}=\left(\begin{array}{cc}1 & 0 \\ 0 & f^{2} g\end{array}\right)$, where $f: R \rightarrow R^{+}$is a warping function, and $g$ is a Riemannian metric on $B$. Let $\tilde{K}$ and $K$ be the curvature tensors of $M$ and $B$, respectively. Then we have $\tilde{K}_{d c b}{ }^{a}=K_{d c b}{ }^{a}-f_{1}^{2}\left(\delta_{d}^{a} g_{c b}-\delta_{c}^{a} g_{d b}\right), \tilde{K}_{1 a b}{ }^{1}=-f f_{11} g_{a b}, \tilde{K}_{b 11}{ }^{a}=-\frac{f_{11}}{f} \delta_{b}^{a}$, and the others are zero, where $f_{1}=\frac{d f}{d t}, f_{11}=\frac{d^{2} f}{d t^{2}}([1,8])$. Hence the Ricci curvature tensors $\tilde{S}$ and $S$ of $M$ and $B$, respectively, are given by

$$
\begin{aligned}
& \tilde{S}_{a b}=S_{a b}-f f_{11} g_{a b}-(n-1) f_{1}^{2} g_{a b}, \\
& \tilde{S}_{a 1}=0, \\
& \tilde{S}_{11}=-\frac{n f_{11}}{f} .
\end{aligned}
$$

The scalar curvatures $\tilde{r}$ and $r$ of $M$ and $B$, respectively, are related by $\tilde{r}=\frac{r}{f^{2}}-\frac{2 n f_{11}}{f}-\frac{n(n-1) f_{1}^{2}}{f^{2}}$. 
For any function $h$ on $M$, the covariant derivatives are given by

$$
\begin{aligned}
& \widetilde{\nabla}_{a} \widetilde{\nabla}_{b} h=\nabla_{a} h_{b}+\left(f f_{1} h_{1}\right) g_{a b}, \\
& \widetilde{\nabla}_{a} \widetilde{\nabla}_{1} h=\partial_{a} h_{1}-\frac{f_{1}}{f} h_{a}, \\
& \widetilde{\nabla}_{1} \widetilde{\nabla}_{1} h=\nabla_{1} \nabla_{1} h,
\end{aligned}
$$

where $\tilde{\nabla}$ and $\nabla$ are operators of the covariant derivatives on $M$ and $B$, respectively.

Suppose that $M=R \times_{f} B$ is a gradient Ricci soliton, then we see that

$$
\begin{aligned}
& \tilde{S}_{a b}=\rho \tilde{g}_{a b}-\widetilde{\nabla}_{a} \widetilde{\nabla}_{b} h=\rho f^{2} g_{a b}-\nabla_{a} h_{b}-f f_{1} h_{1} g_{a b}, \\
& \tilde{S}_{1 a}=\rho \tilde{g}_{1 a}-\widetilde{\nabla}_{1} \widetilde{\nabla}_{a} h=-\partial_{1} h_{a}+\frac{f_{1}}{f} h_{a}, \\
& \tilde{S}_{11}=\rho \tilde{g}_{11}-\widetilde{\nabla}_{1} \widetilde{\nabla}_{1} h=\rho-\nabla_{1} \nabla_{1} h,
\end{aligned}
$$

where $\rho$ is constant on $M$ and $h_{1}=\frac{d h}{d t}$. Using (3.1) and (3.3), we obtain

$$
\begin{aligned}
S_{a b}-f f_{11} g_{a b}-(n-1) f_{1}^{2} g_{a b} & =\rho f^{2} g_{a b}-\nabla_{a} h_{b}-f f_{1} h_{1} g_{a b}, \\
\partial_{1} h_{a} & =\frac{f_{1}}{f} h_{a}, \\
\nabla_{1} \nabla_{1} h & =\rho+\frac{n f_{11}}{f} .
\end{aligned}
$$

Consider the case that $f_{11}(t) \neq 0$ for the warping function $f$. Assume that $h_{a}=\frac{\partial h}{\partial v_{a}} \neq 0$, where $a=2,3, \ldots, n+1$. Then from $(3.4)_{2}$, we get

$$
\frac{\partial}{\partial t}\left(\ln h_{a}\right)=\frac{\partial_{t} h_{a}}{h_{a}}=\frac{f_{1}}{f}=\frac{d}{d t}(\ln f) .
$$

Hence we successively obtain $\ln h_{a}=\ln f+\ell\left(v_{2}, \ldots, v_{n+1}\right), h_{a}=f e^{\ell\left(v_{2}, \ldots, v_{n+1}\right)}$, $h=f \int e^{\ell\left(v_{2}, \ldots, v_{n+1}\right)} d v_{a}$, and

$$
\frac{\partial^{2} h}{\partial t^{2}}=f_{11}(t) \int e^{\ell\left(v_{2}, \ldots, v_{n+1}\right)} d v_{a} .
$$

From $(3.4)_{3}$ and the fact that $\nabla_{1} \nabla_{1} h=\frac{\partial^{2} h}{\partial t^{2}}$, we see that $\frac{\partial^{2} h}{\partial t^{2}}$ depends only on $t$. Henceforth the right-hand side of the equation (3.6) also depends only on $t$. In this case, $\int e^{\ell\left(v_{2}, \ldots, v_{n+1}\right)} d v_{a}$ is either constant or a function of $t$. But this is impossible. Hence we get $\frac{\partial h}{\partial v_{a}}=0$. From this fact and $(3.4)_{1}$, the Ricci curvature $S$ on $B$ becomes

$$
S_{a b}=\left\{f f_{11}+(n-1) f_{1}^{2}+\rho f^{2}-f f_{1} h_{1}\right\} g_{a b} \equiv A g_{a b} .
$$

Since $\frac{\partial h}{\partial v_{a}}=0$, the function $\frac{\partial A}{\partial v_{a}}=0$. So $A$ is constant on $B$. This means that $B$ is an Einstein space. Therefore we have:

Theorem 3.1. Let $M=R \times_{f} B$ be a gradient Ricci soliton and $f_{11}(t) \neq 0$. Then $B$ is an Einstein space. 
Next consider the case $f_{11}(t)=0$. Then $f$ is of the form $f(t)=a t+b$, where $a$ and $b$ are constants. Since $f$ is positive on $R, f$ should be of the form $f=b(>0)$. Then $M$ is the Riemannian product of $R$ and $B$. Hence we can state:

Theorem 3.2. Let $M=R \times_{f} B$ be a gradient Ricci soliton and $f_{11}(t)=0$. Then $B$ is a gradient Ricci soliton.

In the warped product space $M=R \times_{f} B$ with a gradient Ricci soliton, if $f_{11} \neq 0$, then the base space $B$ is Einstein from Theorem 3.1. Due to Theorem 3.2 , we see that $B$ is a gradient Ricci soliton when $f_{11}=0$, but we can't decide whether $B$ is Einstein or non-Einstein.

Consider the space $M=R \times{ }_{c} S^{n}(k)$, where $S^{n}(k)$ is the $n$-sphere with radius $k$. Then $M$ is a gradient Ricci soliton but non-Einstein by use of (3.1). Hence we have:

Example 3.3. $R \times_{c} S^{n}(k)$ is a non-Einstein gradient Ricci soliton with an Einstein leaf $S^{n}(k)$.

Consider the space $N=R \times\left(R \times{ }_{c} S^{n}(k)\right)$. Then $N$ is a gradient Ricci soliton, but $R \times{ }_{c} S^{n}(k)$ is not an Einstein space. Thus we can state:

Example 3.4. The space $N=R \times\left(R \times{ }_{c} S^{n}(k)\right)$ is a gradient Ricci soliton but $R \times{ }_{c} S^{n}(k)$ is not an Einstein space.

In conclusion, Theorems 3.1 and 3.2 give a criterion whether the base space $B$ is Einstein or a gradient Ricci soliton in the total space $R \times_{f} B$ through the calculation of $f_{11}$.

\section{Gradient Ricci solitons in Lorentzian warped product spaces}

The Lorentzian metric in the warped product space $M=R \times_{f} B$ is given by $\tilde{g}=\left(\begin{array}{cc}-1 & 0 \\ 0 & f^{2} g\end{array}\right)$, where $f: R \rightarrow R^{+}$is a warping function, and $g$ is a Riemannian metric on $B$. Then we can see that $\left\{\begin{array}{c}\overline{1} \\ a b\end{array}\right\}=f f_{1} g_{a b},\left\{\begin{array}{c}a \\ b 1\end{array}\right\}=\frac{f_{1}}{f} \delta_{b}^{a}$, $\left\{\begin{array}{c}a \\ b c\end{array}\right\}=\left\{\begin{array}{c}a \\ b c\end{array}\right\}$, and the others are zero, where the range of indices $a, b, c, \ldots$ is $\{2,3, \ldots, n+1\}$.

The curvature $\tilde{K}$ and $K$ of $M$ and $B$ are given by $\tilde{K}_{d c b}{ }^{a}=K_{d c b}{ }^{a}+$ $f_{1}^{2}\left(\delta_{d}^{a} g_{c b}-\delta_{c}^{a} g_{d b}\right), \tilde{K}_{1 a b}{ }^{1}=f f_{11} g_{a b}, \tilde{K}_{b 11}{ }^{a}=-\frac{f_{11}}{f} \delta_{b}^{a}$, and the others are zero. Hence the Ricci curvature tensors $\tilde{S}$ and $S$ of $M$ and $B$, respectively, are reduced to

$$
\begin{aligned}
\tilde{S}_{a b} & =S_{a b}+f f_{11} g_{a b}+(n-1) f_{1}^{2} g_{a b}, \\
\tilde{S}_{a 1} & =0 \\
\tilde{S}_{11} & =-\frac{n f_{11}}{f},
\end{aligned}
$$


and the scalar curvatures $\tilde{r}$ and $r$ of $M$ and $B$, respectively, are related by $\tilde{r}=\frac{r}{f^{2}}+\frac{2 n f_{11}}{f}+\frac{n(n-1) f_{1}^{2}}{f^{2}}$.

Hence, for any function $h$ on $M$, the covariant derivatives are given by

$$
\begin{aligned}
& \widetilde{\nabla}_{a} \widetilde{\nabla}_{b} h=\nabla_{a} h_{b}-\left(f f_{1} h_{1}\right) g_{a b}, \\
& \widetilde{\nabla}_{a} \widetilde{\nabla}_{1} h=\partial_{a} h_{1}-\frac{f_{1}}{f} h_{a}, \\
& \widetilde{\nabla}_{1} \widetilde{\nabla}_{1} h=\nabla_{1} \nabla_{1} h,
\end{aligned}
$$

where $\tilde{\nabla}$ and $\nabla$ are operators of the covariant derivatives on $M$ and $B$, respectively.

If $M=R \times_{f} B$ is a gradient Ricci soliton, then we obtain

$$
\begin{aligned}
& \tilde{S}_{a b}=\rho \tilde{g}_{a b}-\widetilde{\nabla}_{a} \widetilde{\nabla}_{b} h=\rho f^{2} g_{a b}-\nabla_{a} h_{b}+f f_{1} h_{1} g_{a b}, \\
& \tilde{S}_{1 a}=\rho \tilde{g}_{1 a}-\widetilde{\nabla}_{1} \widetilde{\nabla}_{a} h=-\partial_{1} h_{a}+\frac{f_{1}}{f} h_{a}, \\
& \tilde{S}_{11}=\rho \tilde{g}_{11}-\widetilde{\nabla}_{1} \widetilde{\nabla}_{1} h=-\rho-\nabla_{1} \nabla_{1} h,
\end{aligned}
$$

where $\rho$ is constant on $M$.

By use of (4.1) and (4.3), we obtain

$$
\begin{aligned}
S_{a b}+f f_{11} g_{a b}+(n-1) f_{1}^{2} g_{a b} & =\rho f^{2} g_{a b}-\nabla_{a} h_{b}+f f_{1} h_{1} g_{a b}, \\
\partial_{1} h_{a} & =\frac{f_{1}}{f} h_{a}, \\
\nabla_{1} \nabla_{1} h & =-\rho+\frac{n f_{11}}{f} .
\end{aligned}
$$

At first, let us consider the case that $f_{11}(t) \neq 0$ for the warping function $f$. Assume that $h_{a}=\frac{\partial h}{\partial v_{a}} \neq 0$. Then from $(4.4)_{2}$, we get

$$
\frac{\partial}{\partial t}\left(\ln h_{a}\right)=\frac{\partial_{t} h_{a}}{h_{a}}=\frac{f_{1}}{f}=\frac{d}{d t}(\ln f) .
$$

Hence we successively obtain $\ln h_{a}=\ln f+\ell\left(v_{2}, \ldots v_{n+1}\right), h_{a}=f e^{\ell\left(v_{2}, \ldots, v_{n+1}\right)}$, $h=f \int e^{\ell\left(v_{2}, \ldots, v_{n+1}\right)} d v_{a}$ and

$$
\frac{\partial^{2} h}{\partial t^{2}}=f_{11}(t) \int e^{\ell\left(v_{2}, \ldots, v_{n+1}\right)} d v_{a} .
$$

Thus using (4.4) 3 and the fact that $\nabla_{1} \nabla_{1} h=\frac{\partial^{2} h}{\partial t^{2}}$, we see that $\frac{\partial^{2} h}{\partial t^{2}}$ depends only on $t$. Henceforth the right-hand side of the equation (4.6) also depends only on $t$. So, $\int e^{\ell\left(v_{2}, \ldots, v_{n+1}\right)} d v_{a}$ is either a constant or a function of $t$. But this is impossible. Hence we get $\frac{\partial h}{\partial v_{a}}=0$. From this fact and $(4.4)_{1}$, the Ricci curvature $S$ on $B$ becomes

$$
S_{a b}=\left\{-f f_{11}+(n-1) f_{1}^{2}+\rho f^{2}+f f_{1} h_{1}\right\} g_{a b} \equiv D g_{a b} .
$$


Since $\frac{\partial h}{\partial v_{a}}=0$, the function $\frac{\partial D}{\partial v_{a}}=0$. So $D$ is constant on $B$. This means that $B$ is an Einstein space. Therefore we have:

Theorem 4.1. Let Lorentzian warped product space $M=R \times_{f} B$ be a gradient Ricci soliton and $f_{11}(t) \neq 0$. Then $B$ is an Einstein space.

Lemma 4.2. If $R \times B$ is the Lorentzian product space, then $B$ is a gradient Ricci soliton.

Proof. By the similar method in (2.1) and (2.2) with $m(t)=-\frac{1}{2} \rho t^{2}+\mu t+\nu$, we can prove the lemma.

Next, assume that $f_{11}(t)=0$. Then $f$ is of the form $f(t)=a t+b$, where $a$ and $b$ are constants. By the same method of the proof of Theorem 3.2, $f$ should be of the form $f=b(>0)$. Then $M=R \times_{f} B$ is a Lorentzian product space $M=R \times{ }_{b} B$. From this fact and Lemma 4.2 , we see that $B$ is a gradient Ricci soliton. Hence we have:

Theorem 4.3. Let Lorentzian warped product space $M=R \times_{f} B$ be a gradient Ricci soliton and $f_{11}(t)=0$. Then $B$ is a gradient Ricci soliton.

By the analogous argument of the Riemannian case in Chapter 3, we have:

Example 4.4. The space $R \times{ }_{c} S^{n}(k)$ with a Lorentzian metric is a non-Einstein gradient Ricci soliton with an Einstein leaf $S^{n}(k)$.

Example 4.5. The space $N=R \times\left(R \times{ }_{c} S^{n}(k)\right)$ is a gradient Ricci soliton, but $R \times{ }_{c} S^{n}(k)$ is not an Einstein space.

\section{References}

[1] A. Besse, Einstein Manifolds, Springer-Verlag, Berlin, 1987.

[2] H. D. Cao, Geometry of Ricci solitons, Lecture note, Lehigh Univ., 2008.

[3] M. Eminenti, G. La Nave, and C. Mantegazza, Ricci solitons: the equation point of view, Manuscripta Math. 127 (2008), no. 3, 345-367.

[4] M. Fernández-López and E. García-Río, A remark on compact Ricci solitons, Math. Ann. 340 (2008), no. 4, 893-896.

[5] R. S. Hamilton, The Ricci flow on surfaces, Mathematics and general relativity (Santa Cruz CA, 1986), 237-262, Contemp. Math. 71, American Math. Soc., 1988.

[6] T. Ivey, Ricci solitons on compact three-manifolds, Differential Geom. Appl. 3 (1993), no. 4, 301-307.

[7] - New examples of complete Ricci solitons, Proc. Amer. Math. Soc. 122 (1994), no. $1,241-245$

[8] B. H. Kim, Warped products with critical Riemannian metric, Proc. Japan Acad. Ser. A Math. Sci. 71 (1995), no. 6, 117-118.

[9] G. Perelman, Ricci flow with surgery on three manifolds, arXiv:math. DG/0303109.

[10] P. Petersen and W. Wylie, On gradient Ricci solitons with symmetry, Proc. Amer. Math. Soc. 137 (2009), no. 6, 2085-2092.

[11] R. Pina and K. Tenenblat, On solutions of the Ricci curvature equation and the Einstein equation, Israel J. Math. 171 (2009), 61-76. 
BYUNG HAK KIM

Department of Applied Mathematics

KYUNG HeE UNIVERSITY

YoNGIN 446-701, KoreA

E-mail address: bhkim@khu.ac.kr

SANG DEOK LEE

Department of Applied Mathematics

DANKOOK UNIVERSITY

CheOnan 330-714, Korea

E-mail address: sdlee@dankook.ac.kr

JiN Hyuk CHOI

Humanitas College

Kyung HeE University

YONGIN 446-701, KorEA

E-mail address: jinhchoi@khu.ac.kr

YOUNG OK LeE

Department of Mathematics

Kyung HeE University

YONGIN 446-701, KorEA

E-mail address: ylee6604@korea.com 